\title{
Prophylactic Ligature of AV Fistula Prevents High Output Heart Failure after Kidney Transplantation
}

\author{
Patrick Hetz $^{a} \quad$ Markus Pirklbauer ${ }^{a} \quad$ Silvana Müller ${ }^{b} \quad$ Lydia Posch $^{c}$ \\ Maria Gummerer ${ }^{c}$ Martin Tiefenthaler ${ }^{a}$ \\ aDepartment of Internal Medicine IV - Nephrology and Hypertension, Medical University Innsbruck, Innsbruck, Austria; \\ bepartment of Internal Medicine III - Cardiology and Angiology, Medical University Innsbruck, Innsbruck, Austria; \\ 'Department for Surgery, University Hospital for Vascular Surgery, Medical University Innsbruck, Innsbruck, Austria
}

\author{
Keywords \\ High-flow fistula · Fistula ligature $\cdot$ Heart failure $\cdot$ Kidney \\ transplantation
}

\begin{abstract}
Background: Arteriovenous (AV) fistulas are considered the gold standard for ensuring safe and long-term vascular access in patients with haemodialysis-dependent end-stage renal disease. However, previous studies demonstrated that high-flow AV fistulas might add additional cardiovascular burden in the post-transplant setting, leading to frequent fistula closure in this population. Currently, there is no consensus regarding management of high-flow fistulas in posttransplant patients with stable kidney function. The present randomized controlled trial examines the effect of prophylactic AV fistula closure on high-output heart failure. Methods: Twenty-eight kidney transplant patients with stable graft function, absence of pre-existing severe cardiac failure, and brachial arterial flow rate of at least 1,500 mL/min were recruited and randomized in a 1:1 ratio to an intervention and control group, respectively. The intervention group was subject to immediate fistula ligature. Patients within the control group were referred to fistula ligature only if the
\end{abstract}

\begin{tabular}{ll}
\hline KARGER & $\begin{array}{l}\text { (c) } 2020 \text { The Author(s) } \\
\text { Published by S. Karger AG, Basel }\end{array}$ \\
karger@karger.com & This is an Open Access article licensed under the Creative Commons \\
www.karger.com/ajn & $\begin{array}{l}\text { Attribution-NonCommercial-4.0 International License (CC BY-NC) } \\
\text { (http://www.karger.com/Services/OpenAccessicense), applicable to } \\
\text { the online version of the article only. Usage and distribution for com- } \\
\text { mercial purposes requires written permission. }\end{array}$
\end{tabular}

main study endpoint high-output heart failure was reached. The latter was defined by the presence of at least 1 clinical sign (i.e., worsening NYHA score) and at least 2 of the following echocardiographic parameters: diameter of right atrium (major) $>53 \mathrm{~mm}$, right atrium (minor) $>44 \mathrm{~mm}$, inferior vena cava $\geq 21 \mathrm{~mm}$, right pulmonary artery $>20 \mathrm{~mm}$, TAPSE $<16$ $\mathrm{mm}$, systolic pulmonal artery pressure $>40 \mathrm{~mm} \mathrm{Hg}$, and/or left ventricular eccentricity index $<1$. During a 24-month follow-up period, quarterly measurements of kidney function, NT-proBNP, and lactate dehydrogenase as well as a biannual echocardiographic check-up were performed. Results: Highoutput heart failure attributable to high-flow fistula was reported in 5 of 13 control patients (38.5\%), whereas no patient in the intervention group presented with clinical and echocardiographic signs of high-output heart failure during the follow-up period. Thus, prophylactic ligature of highflow fistulas avoided high-output heart failure in our patient population ( $p=0.013$ ). Three patients in the control group, however, had to undergo fistula ligature due to aneurysm formation $(n=2)$ and steal phenomenon $(n=1)$. Median NTproBNP levels decreased from $317 \mathrm{ng} / \mathrm{L}$ pre-ligature to 223 ng/L post-ligature $(p=0.003)$. Serum creatinine levels did not significantly differ before and after AV fistula ligature (1.69 vs. $1.60 \mathrm{mg} / \mathrm{dL}$, respectively, $p=0.059$ ). Improvement 
of echocardiographic findings (e.g., a decrease in systolic pulmonary arterial pressure) was found in 7 of 8 ligature patients but did not reach statistical significance. Conclusion: Prophylactic ligature of high-flow AV fistulas after kidney transplantation can avoid high-output heart failure, and a more liberal approach to close AV fistulas might be justified.

(C) 2020 The Author(s)

Published by S. Karger AG, Basel

\section{Introduction}

Arteriovenous (AV) fistulas are considered the gold standard of long-term vascular access for haemodialysisdependent end-stage renal disease patients based on complication and patency rate $[1,2]$. While AV fistulas are associated with superior outcome compared to AV grafts and central venous catheters, they are still associated with CV burden. Dundon et al. [3] showed a significant increase in left ventricular (LV) and right ventricular end-systolic volume $(21 \%$ rise in the left ventricle and $18 \%$ in the right ventricle) as well as LV mass 6 months after fistula creation. These adaptations are necessary to keep cardiac output stable while enabling an observed rise $(>2,000 \%)$ in brachial artery flow. Post-transplant AV fistula management is of particular interest, though there is no consensus so far. Studying renal transplant patients with patent $(n=39)$ versus closed $(n=22)$ AV fistulas retrospectively, no significant difference in cardiac structure and function was reported between groups [4]. In contrast, Movilli et al. [5] presented favourable effects of $\mathrm{AV}$ fistula ligature on LV hypertrophy in a prospective trial including 61 haemodialysis patients. We previously demonstrated that the necessity of AV fistula ligation is higher than discussed in the literature [6]: In this retrospective study involving 113 patients, 29 patients (i.e., 25.7\%) had to undergo ligature of AV fistula after successful renal transplantation. The predominant reason (53\%, i.e., 15 patients) for fistula closure was symptomatic heart failure with echocardiographic pathology of the right heart. After AV fistula closure, clinical symptoms and capacity for daily-life activities significantly improved in all patients. Remarkably, mean fistula flow rate in patients with and without the need for AV fistula ligation was substantially different, with 2,300 and $850 \mathrm{~mL} /$ min, respectively. Hicks et al. [7] analyzed the incidence of fistula ligature in US transplantation centres using the USRDS database. In a total of 16,845 patients with patent AV fistula or patent AV graft, only $4.6 \%$ (i.e., 779 patients) underwent fistula closure. There was a great difference in between the 248 participating transplant cen- tres. In $>43 \%$ of hospitals, no single ligature was made; however, $11 \%$ of the clinics performed ligation in $>10 \%$ of transplanted patients. Weekers et al. [8] report rapid decline of eGFR after AV fistula closure and recommend to avoid fistula closing. Other studies focused on fistularelated development of pulmonary hypertension (PAH) which is often caused by elevated pulmonary blood flow and/or increased pulmonary vascular resistance [9-11]. $\mathrm{PAH}$ is generally more frequent in patients on haemodialysis $(31.6 \%)$ compared with patients on peritoneal dialysis $(8.3 \%)$ or after kidney transplantation (5\%). Remodelling of the pulmonary vascular system seems to be reversible after renal transplantation. This remodelling may be blocked or PAH may even deteriorate in patients with high-flow fistulas. In this regard, 2 case studies by Clarkson et al. [12] and Kabitz et al. [13] showed that elevated systolic pulmonary artery pressure may dissolve immediately after fistula ligature. By reviewing this controversial topic, Vanderweckene et al. [14] demonstrate an enduring need for more prospective data on long-term effects of fistula ligature on morbidity and mortality in kidney transplant patients. The aim of our study was to test whether prophylactic post-transplant ligature of AV fistulas could prevent the occurrence of adverse cardiac remodelling and consecutive heart failure related to high fistula flow in a randomized controlled intervention trial.

\section{Material and Methods}

This prospective randomized controlled trial was conducted at the Department of Internal Medicine IV, Nephrology and Hypertension, Medical University Innsbruck. Approval from the local ethics committee was obtained prior to study initiation. Written informed consent was obtained from all patients before enrolment.

Kidney transplant patients with regular post-transplant followup at the Department of Internal Medicine IV were eligible for study participation when meeting the following inclusion criteria:

- Kidney transplantation in the past 3-24 months at the Department of Surgery, Medical University Innsbruck.

- AV fistula with a brachial artery flow of at least $1,500 \mathrm{~mL} / \mathrm{min}$ assessed with ultrasound flow measurement. Stable renal graft function (i.e., eGFR of $>30 \mathrm{~mL} / \mathrm{min}$ and eGFR decline $<10 \mathrm{~mL} /$ min during months 3-6 after transplantation).

Exclusion criteria were pre-existing heart failure with ejection fraction of less than $25 \%$, sPAP $>40 \mathrm{~mm} \mathrm{Hg} 3$ months after kidney transplantation, NT-proBNP value of $>1,400 \mathrm{pg} / \mathrm{mL}$, or symptoms of congestive heart failure according to NYHA III or IV.

250 patients were screened between 2013 and 2018 in the context of their regular post-transplantation visits at the Department of Internal Medicine IV (see Fig. 1). Screening included initial echocardiography and Doppler ultrasound-based assessment of fistula flow rate. Doppler ultrasound examination was performed 


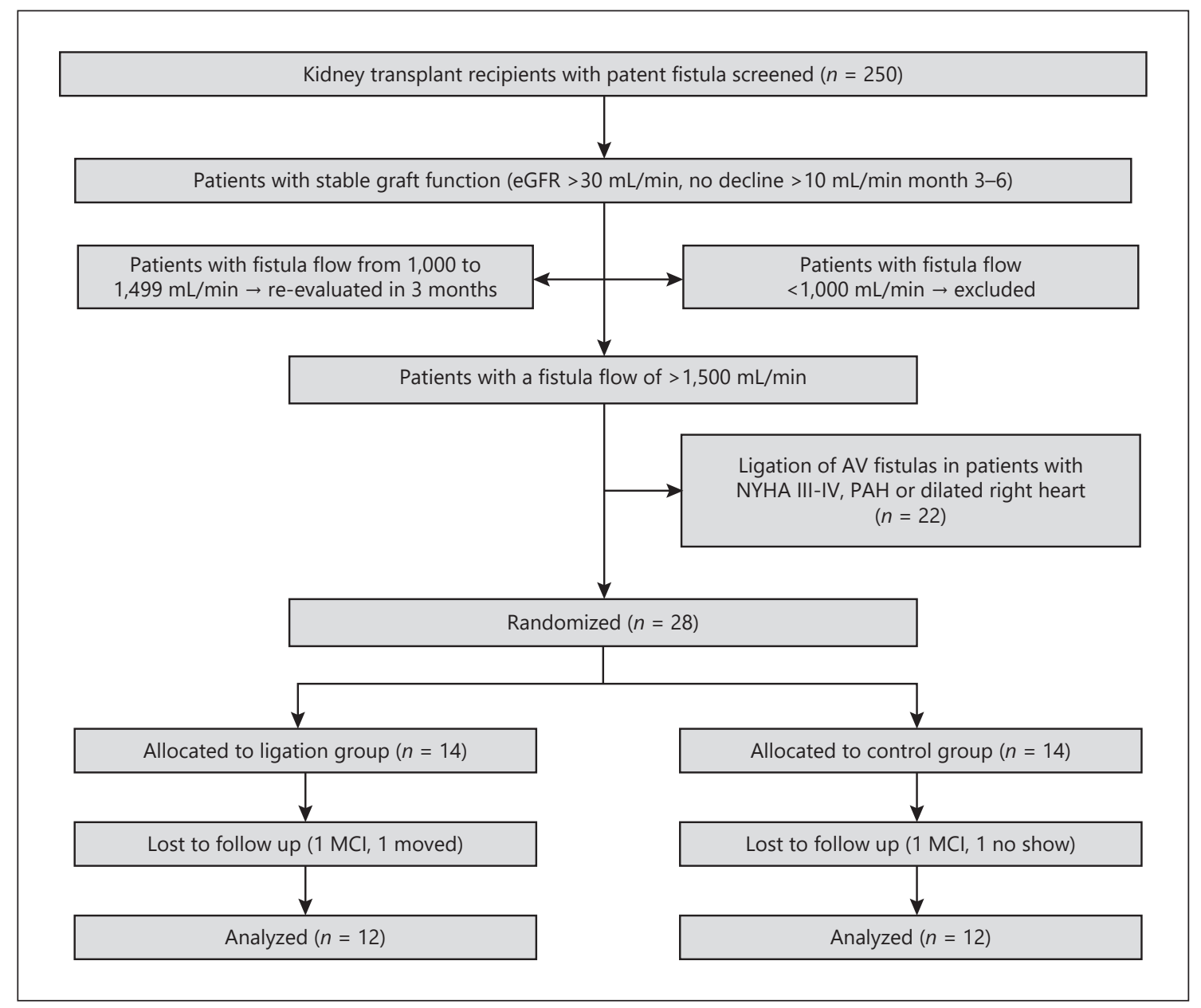

Fig. 1. Screening and allocation algorithm.

by a single nephrologist with the highest experience in sonographic fistula flow examination at the Department of Internal Medicine IV using A Philips iU22 ultrasound device and a L17-5 (17 MHz) linear probe. Time-average velocity of the cross-sectional area of the brachial artery $(\mathrm{AB})$ was assessed proximal of the elbow. The flow volume of the fistula is the calculated product of mean velocity $\times$ cross-sectional area. Mean flow volume rate of 3 (in case of $<10 \%$ inter-measurement variation) to 5 (in case of $10-20 \%$ intermeasurement variation) consecutive measurements was used [15].

Patients were divided into 3 groups according to fistula flow: Group 1: fistula flow $<1,000 \mathrm{~mL} / \mathrm{min}$. Patients assigned to group 1 were excluded because of a low likelihood of developing high-output heart failure. Group 2: fistula flow of 1,000-1,500 $\mathrm{mL} / \mathrm{min}$. Patients assigned to group 2 underwent another fistula flow assessment 3 months after the screening visit and were re-assigned to group 3 if fistula flow increased to $>1,500 \mathrm{~mL} / \mathrm{min}$. Group 3: fistula flow $>1,500 \mathrm{~mL} / \mathrm{min}$ on first ultrasound flow assessment. These subjects were evaluated by echocardiography. Twenty-two patients (16/22 with brachiocephalic fistula) with established PAH or/and right cardiac dilatation at the first echocardiographic assessment were not eligible for randomization and referred to fistula ligation 3 months after transplantation. The remaining 28 pa- tients allocated to group 3 fully met the inclusion criteria and were randomized in a 1:1 ratio to control and intervention groups. While the intervention group was subject to immediate AV fistula ligature at the Department of Vascular Surgery before entering a 24-month follow-up period, the control group entered follow-up without upfront surgical intervention. Both groups received the same accurate follow-up with at least biannually echocardiographic ultrasound examination and fistula flow measurement. Parameters of renal function (i.e., serum creatinine and urea level as well as eGFR according to the MDRD formula [16]) were assessed at least 3 monthly in the context of patients' routine follow-up.

The primary study endpoint was occurrence of right heart failure defined by at least 1 clinical sign and at least 2 echocardiographic parameters suggestive for right heart failure:

- Clinical signs of right heart failure: Dyspnoea on exercise (i.e., worsening in NYHA score), pleural effusions, or peripheral oedema. All patients were encouraged to exercise on a daily basis by walking after renal transplantation and to gradually increase their exercise program. If walking distance did not improve or shortness of breath occurred, patients were subject to further evaluation (i.e., echocardiography, chest X-ray, and laboratory testing). 
Table 1. Patients' characteristics at baseline

\begin{tabular}{lcc}
\hline Characteristics & Intervention $(n=14)$ & Control $(n=14)$ \\
\hline Age, years, median (IQR) [range] & $52.5(15.5)[18-67]$ & $52.5(11.5)[31-69]$ \\
Sex, $n$ (\%) & $12(85.7)$ & $12(85.7)$ \\
$\quad$ Male & $2(14.3)$ & $2(14.3)$ \\
$\quad$ Female & $34(24.5)[14-90]$ & $32.5(32.75)[5-74]$ \\
Dialysis time, months (IQR) [range] & $0.98(0.25)[0.78-1.4]$ & $1.00(0.58)[0.69-1.57]$ \\
Fistula flow, L/min per m ${ }^{2}$ body surface, median (IQR) [range] & $1.85(0.48)[1.59-2.75]$ & $1.8(0.67)[1.5-2.8]$ \\
Fistula flow, L/min, median (IQR) [range] & $3(21.4)$ & $6(42.9)$ \\
Proportion of upper arm fistulas, $n$ (\%) & $45(13.76)$ & $43(10.77)$ \\
eGFR, mL/min, mean (SD) & $1.64(0.62)$ & $1.69(0.47)$ \\
Creatinine, mg/100 mL, mean (SD) & $317(864)$ & $281(166)$ \\
NT-proBNP, ng/L, median (IQR) & $30.5(16.7)$ & $35(11.8)$ \\
Systolic PAP, mm Hg, median (IQR) & $36.5(8.25)$ & $38(11)$ \\
Diameter right atrium minor, mm, median (IQR) & $46.5(6.45)$ & $48(14)$ \\
Diameter right atrium major, mm, median (IQR) & &
\end{tabular}

- Echocardiographic criteria for right heart failure: Diameter of right atrium major $>53 \mathrm{~mm}$, diameter of right atrium minor $>44 \mathrm{~mm}$, inferior vena cava $\geq 21 \mathrm{~mm}$, diameter of right pulmonary artery $>20 \mathrm{~mm}$, TAPSE $<16 \mathrm{~mm}$, systolic pulmonal artery pressure $>40 \mathrm{~mm} \mathrm{Hg}$, and left ventricular eccentricity index $<1$. Echocardiographic monitoring was performed at the Department of Cardiology at MUI. Echocardiographic criteria were established by our on-site cardiologist, who performed or supervised all echocardiographic exams assuring the smallest inter-operator difference for each parameter. Measurement of right atrial and ventricular diameter as well as the pulmonal artery pressure was conducted according to standard operating procedures. Measurement of systolic pulmonal artery pressure followed the principle of pulmonal outflow tract acceleration time [17].

In addition to planned biannual echocardiographic examination, patients presenting with clinical signs of heart failure were immediately referred to additional echocardiographic examination. Patients fulfilling echocardiographic and clinical criteria of right heart failure in the control group were immediately subject to fistula closure. According to state-of-the-art literature, AV fistula ligature is indicated in patients with clinical and echocardiographic signs of high-output heart failure and stable kidney graft function. Thus, remaining below echocardiographic criteria of right heart failure (see the definition earlier) was a prerequisite for entering the study. The majority of patients with upper arm fistula, especially women, had to be primarily excluded from this study due to these criteria. Pre-existing minor elevation in pulmonal artery pressure $(<40 \mathrm{~mm} \mathrm{Hg})$ or pre-existing single right ventricular dilatation was no exclusion criteria since elevated PAH levels are common in patients with haemodialysis and after recent transplantation $[10,11]$. However, in case of pre-existing minor $\mathrm{PAH}$ or single right ventricular dilatation, cautious screening for deterioration of pulmonary artery pressure or right ventricular dilatation was conducted. For patients with a single pre-existing right ventricular dilatation, a diameter increase of $50 \%$, an ejection fraction of $<25 \%$, or an NT-proBNP of $>1,600 \mathrm{ng} / \mathrm{mL}$ during follow-up prompted surgical AV fistula ligation. Focussing on right heart pathologies has been based on the available literature examining the right heart system in nephrological context ("renal fistula toxicity") [18-20]. In this regard, Floccari et al. [20] showed that a reduction in compliance and systolic function of right heart chambers occurs early and frequently in the course of chronic kidney disease. In addition to assessing between-group differences in the occurrence of right heart failure (i.e., primary end point), we investigated the change in NT-proBNP levels, pulmonary artery pressure, diameter of right atrium, and renal function parameters after fistula ligature. Body-surface calculation was done according to the Mosteller formula [21]. Patient follow-up was maintained for 24 months after randomization. Upon completion of followup, most patients continued post-transplant care at our department.

\section{Creation and Ligation of Autogenous AV Fistulas}

The following techniques are common to all autogenous vein access creations in our department of vascular surgery: The distal end of the vein is transected and ligated. After clamping the artery, a longitudinal arteriotomy of about $4-\mathrm{mm}$ length is made. The AV anastomosis is performed end to side with a 6-0 or 7-0 monofilament non-absorbable continuous suture.

For ligation, the vein will be dissected, mobilized, and transected between two clamps close to the anastomosis. After ligating the proximal end, the artery will be reconstructed with a linear suture. In case of vessel elongation, the anastomosis will be resected completely and an end-to-end anastomosis of the artery will be performed. Venous aneurysm is resected close to the anastomosis, and the remaining vein is left in situ. To obtain better cosmetic results and to reduce the risk of developing thrombosis of aneurysmatic fistulas, we apply a postoperative arm compression bandage for 3 weeks.

\section{Statistical Analysis}

Clinical data collection was performed using routinely implemented computerized clinical documentation systems at our department (Powerchart and Diadem). The distribution of high-output heart failure between groups was analyzed using the chi-quadrat test based on $2 \times 2$ tabulation. The comparison of laboratory 
Table 2. Ligature criteria in patients (control group)

\begin{tabular}{|c|c|c|c|}
\hline Patient 1 & \multicolumn{3}{|c|}{ Systolic PAP $50 \mathrm{~mm} \mathrm{Hg}$, diameter of right atrium 40/53 mm, RPA $19 \mathrm{~mm}$ (borderline to $20 \mathrm{~mm}$ ) } \\
\hline \multirow[t]{2}{*}{ Patient 2} & \multicolumn{3}{|c|}{$\begin{array}{l}\text { Systolic PAP } 40 \mathrm{~mm} \mathrm{Hg} \text {, RPA } 23 \mathrm{~mm} \text {, diameter of right atrium } 49 / 58 \mathrm{~mm} \text { (fulfilling both criteria for right atrium } \\
\text { dilatation), TAPSE } 13\end{array}$} \\
\hline & \multicolumn{3}{|c|}{ Patient's condition: newly occurred dyspnoea NYHA III } \\
\hline \multirow[t]{2}{*}{ Patient 4} & \multicolumn{3}{|c|}{ Diameter of right atrium 45/55 mm (fulfilling both criteria for right atrium dilatation) } \\
\hline & \multicolumn{3}{|c|}{ Patient's condition: newly occurred dyspnoea NYHA II with very high shunt volume (2.8 L/min) } \\
\hline \multirow[t]{2}{*}{ Patient 5} & \multicolumn{3}{|c|}{ RPA $21 \mathrm{~mm}$, diameter right atrium $44 / 49 \mathrm{~mm}$, left ventricular eccentricity index 0.84 , Inferior vena cava $>21 \mathrm{~mm}$} \\
\hline & \multicolumn{3}{|c|}{ Patient's condition: worsening leg oedema with very high shunt volume $(2.8 \mathrm{~L} / \mathrm{min})$} \\
\hline \multicolumn{2}{|c|}{ Characteristics } & $\begin{array}{l}\text { Intervention group } \\
\text { patients, } n=14\end{array}$ & $\begin{array}{l}\text { High-output failure } \\
\text { patients, } n=5\end{array}$ \\
\hline \multicolumn{2}{|c|}{ Time between study recruitment and ligature, months (range) } & $1.0(1-8)$ & $7.0(4-24)$ \\
\hline \multicolumn{2}{|c|}{ Time on dialysis (range) } & $34.0(14-90)$ & $43.0(12-74)$ \\
\hline \multicolumn{2}{|c|}{ Diameter of right atrium major, mm, median (range) } & $46.5(36-52)$ & $53(53-58)$ \\
\hline \multicolumn{2}{|c|}{ Diameter of right atrium minor, mm, median (range) } & $36.5(29-42)$ & $45(40-49)$ \\
\hline \multicolumn{2}{|c|}{ Median systolic PAP, mmHg, median (range) } & $30,5(25-50)$ & $35(27-50)$ \\
\hline \multicolumn{2}{|c|}{ NT-proBNP, ng/L, median (range) } & $317(50-1907)$ & $337(134-2239)$ \\
\hline \multicolumn{2}{|c|}{ Creatinine level, mg/100 mL, mean (range) } & $1.64(0.96-3.11)$ & $1.83(1.32-2.27)$ \\
\hline
\end{tabular}

and echocardiographic findings before and after fistula ligature was performed using a 2-tailed $t$ test for normal distributed data or the Wilcoxon signed-rank test for non-normal distributed data. Normal distribution of data was assessed by using the ShapiroWilk test. Correlation between fistula flow rate and various clinical parameters was conducted by using linear regression analysis and ANOVA. The cut-off for statistical significance was set at a $p$ value of $<0.05$. Statistical analysis was performed using Microsoft Excel and SPSS.

\section{Results}

The baseline characteristics of intervention and control group patients are shown in Table 1 . In each group, 1 patient died during the follow-up period, but deaths were not related to the study procedure. In the intervention group, 1 patient died of suspected myocardial infarction 5 months after fistula ligature. In the control group, death was confirmed as myocardial infarction related. No renal- or heart-failure-related deaths were observed. In each group, 1 patient was lost to follow-up.

The primary endpoint right heart failure, and thus indication for fistula ligature, occurred in $38.5 \%$, i.e., in 5 of 13 control group patients (per-protocol analysis). In the intervention group, no patient presented with right heart failure according to study definitions. Prophylactic AV fistula ligature prevented right heart failure compared to the control group $(p=0.013)$. The clinical presentation of each patient meeting ligature criteria is shown in Table 2. No significant correlation was found between any laboratory parameter and the occurrence of right heart failure in the control group. The predominant criterion for reaching the echocardiographic end point right heart failure was dilatation of the right atrium with a diameter $>53$ $\mathrm{mm}$ major or $>44$ minor $(80 \%)$. Two patients presented with increased systolic PAP, one each with TAPSE $<16$ 
$\mathrm{mm}$, left ventricular eccentricity index abnormalities, or dilated inferior vena cava. Clinical characteristics of patients receiving prophylactic fistula ligature (intervention group) and patients that underwent fistula ligature due to occurrence of right heart failure (control group) are shown in Table 3. No significant between-group differences were found.

Overall, AV fistula ligation led to a decrease in NTproBNP levels when comparing median levels directly before and after fistula closure in all patients that underwent ligature (intervention group + control group). Median NT-proBNP pre-ligature was 317 ng/L (95\% CI \pm 292 ) compared to $223 \mathrm{ng} / \mathrm{L}(95 \% \mathrm{CI} \pm 243)$ post-ligature $(p=0.003)$. Eighteen of 21 patients that underwent ligature ( 2 patients excluded due to missing laboratory data) presented with a fall in NT-proBNP levels (median decrease of 36\%, 95\% CI $\pm 18 \%$ ). Comparing the average of last $3 \mathrm{NT}$-proBNP values before ligature with the average of first 3 values after ligature, the same trend could be observed: Median average levels before and after surgical intervention were $327 \mathrm{ng} / \mathrm{L}$ (95\% CI $\pm 390)$ and $202 \mathrm{ng} / \mathrm{L}(95 \% \mathrm{CI} \pm 219)$, respectively ( $p=$ $0.006)$.

Mean creatinine levels remained stable after fistula ligature in 21 patients ( 2 patients with missing laboratory data), that is, $1.69 \mathrm{mg} / \mathrm{dL}(95 \% \mathrm{CI} \pm 0.27)$ pre-ligature versus $1.60 \mathrm{mg} / \mathrm{dL}(95 \% \mathrm{CI} \pm 0.24)$ post-ligature. $14 \mathrm{pa}-$ tients experienced a drop in serum creatinine levels ranging from -7 to $-28 \%$, and 7 patients presented increasing serum creatinine levels from +1 to $+46 \%$. Overall, a nonsignificant mean creatinine decrease of $7 \%(95 \% \mathrm{CI} \pm 7 \%)$ from pre- to post-ligature was observed $(p=0.059)$. Thus, in our study cohort, the closure of AV fistula seems to have little impact on renal function with a slight tendency towards declining creatinine levels.

The following echocardiographic changes were documented after fistula ligature: The mean diameter of the right atrium (major) showed a slight reduction from 48 $\mathrm{mm}(95 \% \mathrm{CI} \pm 4.2)$ to $45 \mathrm{~mm}(95 \% \mathrm{CI} \pm 2.21)$. Ten of 15 patients with available echo data 3 months after ligature showed a mean decrease in atrium size of $11 \%$ (range 4-45\%). However, observed changes in right heart diameters were not statistically significant.

No patient in the intervention group presented with symptoms of congestive heart failure during the 24-month follow-up period. As in the total cohort of patients that underwent ligature, NT-proBNP levels declined in the intervention group: 9 of 10 patients with available NTproBNP levels directly after ligature showed falling or unchanged NT-proBNP values. The median NT-proBNP value was $510 \mathrm{ng} / \mathrm{L}(95 \% \mathrm{CI} \pm 349)$ pre-OP, compared to $317 \mathrm{ng} / \mathrm{L}(95 \% \mathrm{CI} \pm 278)$ post-OP $(p=0.021)$. Likewise, the average of last 3 NT-proBNP values before and first 3 NT-proBNP values after ligature was 317 and $232.5 \mathrm{ng} / \mathrm{L}$, respectively ( $p$ value 0.037 ). No significant correlation was found between fistula flow rate and NT-proBNP, atrial size, or systolic PAP.

During post-study observation, all 12 intervention group patients ( 1 death and 1 lost to follow-up) remained stable in cardiac and renal function (up to 88 months of routine post-transplantation follow-up). In the intervention group, 7 of 8 patients with available data (i.e., echo within 3 months post-OP) had better or constant systolic PAP levels (87.5\%), 1 patient had an increase in pulmonal artery pressure $(p=0.115)$, and 6 of 7 patients $(85.7 \%)$ showed an improved TAPSE as parameter for right heart function [22] within 3 months after ligature. Along with these echocardiographic findings, all patients reported better performance status, resolving shortness of breath and easing in daily routine activities after fistula ligature. In the control group, 1 patient $(7.15 \%)$ underwent fistula ligation due to exercise-limiting acral ischaemia ("steal phenomenon") and 2 patients (14.3\%) due to progredient aneurysm with progressive risk of rupture. Another patient (7.15\%) underwent fistula ligation because of impaired performance status and deteriorating dyspnoea without meeting echo criteria for right heart failure. The patient recovered quickly after fistula ligation and improved from NYHA class IV to II. AV fistula closure was an overall safe procedure; however, 1 case of bilateral pulmonary embolism was observed after removing fistula compression within 1 week after the surgical intervention. In all other patients where compression was continued for at least 3-4 weeks after fistula closure, no such complication could be observed. In addition, 1 patient developed thrombosis of post-ligature dilated radial artery (diameter 6-8 $\mathrm{mm}$ ) within 5 days after surgical intervention. This intervention-related complication was managed conservatively because of maintained forearm perfusion via a patent and also dilated A. ulnaris (3-4 mm).

\section{Discussion}

There is substantial uncertainty regarding fistula management after successful kidney transplantation. Highflow fistulas are regularly closed when aneurysm with concomitant risk of rupture, steal syndrome with acral ischaemia, and/or congestive heart failure at NYHA stage 
III or IV are present. However, both in the presence and absence of typical clinical ligature indications, there is great variety in routine clinical management [14]. With respect to own retrospective data [6] showing up to $25 \%$ occurrence of high-output heart failure with consecutive closure of fistulas, we conducted a prospective randomized controlled trial evaluating the potential benefit of prophylactic ligature of high-flow AV fistulas. Patients with fistula flow $>1,500 \mathrm{~mL} / \mathrm{min}$, stable renal function, and absence of clinical and echocardiographic signs necessitating urgent fistula ligation were randomized in a 1:1 ratio to an intervention and control group. In the intervention group, patients were subject to immediate AV fistula ligation, and in the control group, patients were cautiously monitored. The occurrence of right heart failure in 5 patients in the control group as compared to no single case in the intervention group confirmed our hypothesis that prophylactic fistula closure prevents highoutput heart failure. We also observed significant reduction of NT-proBNP levels in 18 of 21 patients undergoing fistula ligation - a strong indication that burden on right and left heart systems might be reduced after fistula ligature. The declining systolic PAP in the intervention group implicates a potential role of pulmonary vascular system in maintaining high cardiac output. Systolic PAP was previously found to be higher in patients on haemodialysis compared to peritoneal dialysis or kidney transplant patients and to decline after renal transplantation [10]. Based on these findings, we thus postponed fistula closure for 3 months after transplantation to include only patients without recovery of $\mathrm{PAH}$ following kidney transplantation.

Reversibility of heart rate ("Nicoladoni-Branham sign") [23] and systolic PAP after (sub)total manual compression of high-flow fistulas for 1 to 2 min may deliver additional important information if high cardiac output is fixed or reversible. In this regard, a compression-induced reduction in echocardiographically assessed SPAP has been shown to be predictive for reversibility of PAH after fistula ligation in $80 \%$ of cases [24]. Reduced TAPSE has been previously demonstrated to correlate with systolic PAP and predict CV mortality risk in congestive heart failure patients with stage III chronic kidney disease, adding significant prognostic information to the NYHA stage [20]. In our control group, however, decreased TAPSE was observed in 1 patient only and thus might not be suitable as single parameter for timing high-flow fistula closure. The definition of highflow fistula in the literature remains unclear. Following own data [6], we used a cut-off of $1,500 \mathrm{~mL} / \mathrm{min}$. A pre-

Prophylactic Fistula Ligature after Kidney

Transplantation vious cross-sectional study used a cut-off of $2,000 \mathrm{~mL} /$ min and normalized fistula flow to body height (raise to the power of 2.7), thereby demonstrating predictive potential of fistula flow for the development of right heart failure [23]. Normalizing fistula flow to body surface area or height reflects differences in patient size more accurately but did not affect the results within our patient cohort (data not shown). A significant decrease in LV mass and improvement of cardiac diameters, output, and index as well as NT-proBNP levels have been shown after AV fistula closure in 27 post-transplant patients; however, no specific fistula flow cut-off for ligature referral was reported [25]. Some previous studies claimed little impact of fistulas on cardiac function [4, 26, 27]; however, retrospective design and short follow-up were substantial limitations of these studies. Our findings are in line with other studies demonstrating a positive impact of fistula ligature on cardiac function [28, 29]. It is important to mention that patients with overt $\mathrm{PAH}$ or signs of heart failure (NYHA III and IV) at screening visit were excluded from the present study and subject to fistula closure if the diagnosis was still confirmed 3 months after kidney transplantation. However, in asymptomatic patients without further options for autologous fistula creation and no progression to right heart failure or overt $\mathrm{PAH}$ in echocardiography, fistula flow reduction by means of PTFE tube interposition at the site of AV fistula anastomosis may be an alternative option instead of fistula ligature. AV fistula banding or reconstruction of anastomosis might reduce $\mathrm{PAH}$ and/or high-output heart failure while maintaining fistula patency; however, these approaches are associated with a high risk of periinterventional thrombosis and their efficacy seems to be rather low. At least in our facility, flow reduction approaches with banding or reconstruction are regularly found to be transient and PAH and/or high-output heart failure frequently re-occurs within 12 months. Due to these facts, we decided to surgically ligate AV fistulas within the present study and generally reserve fistula banding or reconstruction of anastomosis for patients without overt heart failure but foreseeable need of dialysis within the next 2 years (e.g., in patients with eGFR $<30$ $\mathrm{mL} / \mathrm{min}$ ). The study's strategy to primarily avoid high fistula flow complications by timely ligation - which seems to be the case according to our study findings might indeed increase the risk of losing fistula options for subsequent treatment of transplant failure; however, during the 24-month follow-up none of the study patients developed dialysis-dependent kidney transplant dysfunction. The cardiovascular benefit of this strategy 
might outweigh any potential risk that comes with a loss of fistula options. In this regard, catheter-based approaches might represent an alternative option for dialysis access. Further large-scale clinical trials are mandatory to assess the risk-benefit ratio of this strategy. Nevertheless, we found that more than half of patients undergoing forearm fistula closure had a venous branch from the dorsal side of the forearm that kept their fistula vein patent after ligation. This might allow to re-use the AV fistula as radiocephalic fistula (proximal forearm) in case of subsequent need of dialysis.

To our knowledge, this is the first prospective, randomized controlled trial showing that prophylactic AV fistula ligature can avoid high-output heart failure in kidney transplant patients with fistula flow $>1,500 \mathrm{~mL} / \mathrm{min}$, stable renal function, and absence of clinical and echocardiographic signs necessitating urgent fistula ligation. Study design and clear-cut definition of the primary endpoint (i.e., right heart failure based on clinical and echocardiographic parameters) are particular study strengths. However, based on its pilot trial character, this singlecentre study has several limitations. In addition to a rather small sample size, echocardiographic examination and NT-proBNP levels were not available within 3 months after ligation in some of the patients. Intra- and interobserver variability was not evaluated with respect to echocardiographic assessment. Nevertheless, despite small sample size, a statistically significant difference in the occurrence of right heart failure was observed between the intervention and control groups. Fistula ligature is generally well tolerated and considered safe with recommended post-interventional compression. Based on the fact that interventional group patients showed declining NT-proBNP and sPAP levels after fistula ligature while maintaining a stable kidney graft function, prophylactic ligature of high-flow fistulas might be justified. In addition to heart failure risk, patients with high-volume fistula flow rates are at increased risk for developing aneurysm and/or steal phenomenon, which highlights the importance of fistula flow monitoring during routine transplant follow-up.

\section{Conclusion}

By demonstrating prevention of right heart failure with prophylactic ligature of high-flow fistulas $(>1,500$ $\mathrm{mL} / \mathrm{min}$ ) in kidney transplant patients with stable graft function, this pilot randomized controlled trial shall encourage further multicentre trial efforts to improve posttransplant fistula management and, thus, patient outcome. Until then, the present study might provide some guidance for clinical management of high-flow fistulas after renal transplantation.

\section{Acknowledgements}

We thank the patients for study participation.

\section{Statement of Ethics}

This prospective randomized controlled trial was conducted at the Department of Internal Medicine IV, Nephrology and Hypertension, Medical University Innsbruck. Approval from the local ethics committee was obtained prior to study initiation. Written informed consent was obtained from all patients before enrolment.

\section{Conflict of Interest Statement}

All authors declare no conflict of interest.

\section{Funding Sources}

The authors declare no funding.

\section{Author Contributions}

M.T.: research idea, study design, patient recruitment, and obtaining ethics committee approval. S.M.: design of echo criteria and performing/supervising echo exams. L.P. and M.G.: surgery of study patients. M.T., S.M., and M.P.: data acquisition. P.H., M.T., and M.P.: data analysis/interpretation. P.H.: statistical analysis. P.H., M.P., M.G., and M.T.: manuscript preparation and drafting. M.T.: responsible for the integrity of data analysis.

\section{References}

1 Ozeki T, Shimizu H, Fujita Y, Inaguma D, Maruyama S, Ohyama Y, et al. The type of vascular access and the incidence of mortality in Japanese dialysis patients. Intern Med. 2017;56(5):481-5.

2 Bae E, Lee H, Kim DK, Oh K-H, Kim YS, Ahn $\mathrm{C}$, et al. Autologous arteriovenous fistula is associated with superior outcomes in elderly hemodialysis patients. BMC Nephrol. 2018 Nov 6;19(1):306.

3 Dundon BK, Torpey K, Nelson AJ, Wong DT, Duncan RF, Meredith IT, et al. The deleterious effects of arteriovenous fistula-creation on the cardiovascular system: a longitudinal magnetic resonance imaging study. Int $\mathrm{J}$ Nephrol Renovasc Dis. 2014;7:337-45.

4 De Lima JJ, Vieira ML, Molnar LJ, Medeiros CJ, Ianhez LE, Krieger EM. Cardiac effects of persistent hemodialysis arteriovenous access in recipients of renal allograft. Cardiology. 1999;92(4):236-9. 
5 Movilli E, Viola BF, Brunori G, Gaggia P, Camerini C, Zubani R, et al. Long-term effects of arteriovenous fistula closure on echocardiographic functional and structural findings in hemodialysis patients: a prospective study. Am J Kidney Dis. 2010 Apr;55(4):682-9.

6 Schier T, Göbel G, Bösmüller C, Gruber I, Tiefenthaler M. Incidence of arteriovenous fistula closure due to high-output cardiac failure in kidney-transplanted patients. Clin Transplant. 2013 Nov-Dec;27(6):858-65.

7 Hicks C, Bae S, Pozo M, DiBrito S, Abularrage $\mathrm{C}$, Segev D, et al. Practice patterns in arteriovenous fistula ligation among kidney transplant recipients in the United States Renal Data Systems. J Vasc Surg. 2019 Sep;70(3): 842-52.e1.

8 Weekers L, Vanderweckene P, Pottel H, Castanares-Zapatero D, Bonvoisin C, Hamoir E, et al. The closure of arteriovenous fistula in kidney transplant recipients is associated with an acceleration of kidney function decline. Nephrol Dial Transplant. 2017 Jan 1;32(1): 196-200.

9 Yigla M, Abassi Z, Reisner SA, Nakhoul F. Pulmonary hypertension in hemodialysis patients: an unrecognized threat. Semin Dial. 2006 Sep-Oct;19(5):353-7.

10 Abedini M, Sadeghi M, Naini AE, Atapour A, Golshahi J. Pulmonary hypertension among patients on dialysis and kidney transplant recipients. Ren Fail. 2013;35(4):560-5.

11 Agarwal AK. Systemic effects of hemodialysis access. Adv Chronic Kidney Dis. 2015 Nov; 22(6):459-65.

12 Clarkson MR, Giblin L, Brown A, Little D, Donohoe J. Reversal of pulmonary hypertension after ligation of a brachiocephalic arteriovenous fistula. Am J Kidney Dis. 2002 Sep; 40(3):e8.1-4.
13 Kabitz HJ, Walterspacher S, Geyer M, Fischer KG, Huber TB, Muendlein E, et al. Reversible pulmonary hypertension in a kidney transplant with patent A-V fistula. Clin Kidney J. 2012 Aug;5(4):347-9.

14 Vanderweckene P, Weekers L, Lancellotti P, Jouret F. Controversies in the management of the haemodialysis-related arteriovenous fistula following kidney transplantation. Clin Kidney J. 2018 Jun;11(3):406-12.

15 Martin AG, Grasty M, Lear PA. Haemodynamics of brachial arteriovenous fistula development. J Vasc Access. 2000 Apr-Jun;1(2): 54-9.

16 Jones GR, Imam SK. Validation of the revised MDRD formula and the original Cockcroft and Gault formula for estimation of the glomerular filtration rate using Australian data. Pathology. 2009;41(4):379-82.

17 Granstam SO, Björklund E, Wikström G, Roos MW. Use of echocardiographic pulmonary acceleration time and estimated vascular resistance for the evaluation of possible pulmonary hypertension. Cardiovasc Ultrasound. 2013 Feb 27;11:7.

18 Nakhoul F, Yigla M, Gilman R, Reisner SA, Abassi Z. The pathogenesis of pulmonary hypertension in haemodialysis patients via arterio-venous access. Nephrol Dial Transplant. 2005 Aug;20(8):1686-92.

19 Amerling R, Ronco C, Kuhlman M, Winchester JF. Arteriovenous fistula toxicity. Blood Purif. 2011;31(1-3):113-20.

20 Floccari F, Granata A, Rivera R, Marrocco F, Santoboni A, Malaguti M, et al. Echocardiography and right ventricular function in NKF stage III chronic kidney disease: ultrasound nephrologists' role. J Ultrasound. 2012 Dec; 15(4):252-6.

21 Mosteller RD. Simplified calculation of bodysurface area. N Engl J Med. 1987 Oct 22; 317(17):1098.
22 Tan Y, Manouras A, Lund L, Venkateshvaran A. Feasibility and accuracy of tricuspid annular displacement assessed by speckle tracking echocardiography and Doppler tissue imaging. Echocardiography. 2019 Nov;36(3):2004-9.

23 Zamboli $\mathrm{P}$, Lucà S, Borrelli S, Garofalo C, Liberti ME, Pacilio M, et al. High-flow arteriovenous fistula and heart failure: could the indexation of blood flow rate and echocardiography have a role in the identification of patients at higher risk? J Nephrol. 2018 Dec;31(6): 975-83.

24 Wasse H, Singapuri MS. High-output heart failure: how to define it, when to treat it, and how to treat it. Semin Nephrol. 2012 Nov; 32(6):551-7.

25 Rao N, Stokes M, Rajwani A, Ullah S, Williams K, King D, et al. Effects of arteriovenous fistula ligation on cardiac structure and function in kidney transplant recipients. Circulation. 2019 Jun 18;139(25):2809-18.

26 Glowinski J, Malyszko J, Glowinska I, Mysliwiec M. To close or not to close: fistula ligation and cardiac function in kidney allograft recipients. Pol Arch Med Wewn. 2012;122(78):348-52.

27 Soleimani M, Shahrokh H, Shadpour P, Shirani $\mathrm{M}$, Arasteh S. Impact of dialysis access fistula on cardiac function after kidney transplantation. Iran J Kidney Dis. 2012 May;6(3): 198-202.

28 van Duijnhoven EC, Cheriex EC, Tordoir JH, Kooman JP, van Hooff JP. Effect of closure of the arteriovenous fistula on left ventricular dimensions in renal transplant patients. Nephrol Dial Transplant. 2001 Feb;16(2): 368-72.

29 Unger P, Wissing KM, de Pauw L, Neubauer $\mathrm{J}$, van de Borne P. Reduction of left ventricular diameter and mass after surgical arteriovenous fistula closure in renal transplant recipients. Transplantation. 2002 Jul 15;74(1):73-9. 